\title{
El derecho comercial: de la regulación al compliance
}

\author{
Commercial Law: From Regulation to Compliance \\ O direito comercial: da regulação à compliance
}

JaVIER SANCLEMENTE-ArCiniEGas ${ }^{*}$

Fecha de ReCePCión: 3 de Junio de 2019. FeCha de APRobación: 19 de octubre de 2019

Doi: https://doi.org/10.12804/revistas.urosario.edu.co/sociojuridicos/a.7958

Para citar: Sanclemente-Arciniegas, J. (2020). El derecho comercial: de la regulación al compliance. Estudios Socio-jurídicos, 22(2), 87-116. Doi: https://doi.org/10.12804/revistas.urosario.edu.co/sociojuridicos/a.7958

\section{RESUMEN}

El presente texto plantea una reflexión sobre las nociones jurídicas del compliance y la regulación económica. Se analiza la manera en la que renuevan viejas instituciones del derecho comercial, como los derechos y las obligaciones de los agentes económicos. Ellas son las herramientas jurídicas de un Estado modesto, pero que pretende seguir siendo el garante de intereses generales. Para ello, desarrolla nuevas formas de controlar tanto el acceso como el comportamiento en el mercado. De esa manera se plantean nuevas tensiones entre el ejercicio del derecho a la libertad económica y la protección del bien común. Dichos conflictos deben tramitarse de modo pragmático, atendiendo la complejidad que se deriva de la mezcla de lógicas diversas. Se observa que las empresas deben armonizar el objetivo de generar utilidades con nuevas obligaciones que les exigen proteger intereses públicos. De esa forma, la responsabilidad social de la empresa adquiere un carácter normativo, pues el incumplimiento de las nuevas obligaciones puede dar lugar a sanciones severas, incluso en materia penal.

Palabras clave: derecho comercial, regulación económica, obligaciones de compliance, conformidad, obligaciones de cumplimiento, acceso al mercado, normalización de la economía, sanciones penales, responsabilidad social de la empresa.

* Doctor en Derecho de la Universidad París-Dauphine, PSL Research University; magíster en Derecho de los Negocios y de la Economía de la Universidad Paris I Pantheón Sorbonne; especialista en Derecho Comercial de la Universidad Paris II Panthéon Assas; especialista en Derecho Público de la Universidad Externado; especialista en Derecho Privado Económico de la Universidad Nacional; abogado de la misma universidad. Ex magistrado auxiliar del Consejo de Estado; actualmente, asesor del Ministerio de Hacienda y Crédito Público, docente de Derecho Económico en la Universidad Nacional de Colombia y miembro del Grupo de Investigación en Derecho y Economía. Correo electrónico: jsanclementea@unal.edu.co. ORCID: https://orcid. org/0000-0002-6251-8177 


\section{ABSTRACT}

This paper proposes a reflection on the legal notions of compliance and economic regulation. It analyzes how old commercial law institutions as the rights and obligations of economic agents are renewed. They are the legal tools of a modest state, which intends to continue being the guarantor of general interests. To do this, it develops new ways to control both access and behavior in the market. In this way, new tensions arise between the exercise of the right to economic freedom and the protection of the common good. These conflicts must be handled in a pragmatically, taking into account the complexity that derives from the mixture of different logics. It is observed that companies must harmonize the objective of generating profits with new obligations that require them to protect public interests. In this way, the company's social responsibility acquires a normative character, since the breach of the new obligations can lead to severe sanctions, even of criminal nature.

Keywords: Commercial law economic regulation, compliance obligations of compliance, market access, normalization of the economy, criminal sanctions social responsibility of the company.

\section{RESUMO}

O presente texto apresenta uma reflexão sobre as noções jurídicas de compliance e regulação econômica. Se analisa a maneira na que renovam velhas instituições do direito comercial como os direitos e as obrigações dos agentes econômicos. Elas são as ferramentas jurídicas de um estado modesto, mas que pretende seguir sendo o garante de interesses gerais. Para sito, desenvolve novas formas de controlar tanto o acesso quanto o comportamento no mercado. Dessa maneira se apresentam novas tensões entre o exercício do direito à liberdade econômica e a proteção do bem comum. Ditos conflitos devem tramitar-se de forma pragmática, atendendo a complexidade que se deriva da mistura de lógicas diversas. Se observa que as empresas devem harmonizar o objetivo de gerar utilidades com novas obrigações que lhes exigem proteger interesses públicos. Desta maneira, a responsabilidade social da empresa adquire um carácter normativo, pois o incumprimento das novas obrigações pode dar lugar a sanções severas, inclusive em matéria penal.

Palavras-chave: direito comercial, regulação econômica, obrigações de compliance, conformidade, obrigações de cumprimento, acesso ao mercado, normalização da economia, sanções penais, responsabilidade social da empresa. 


\section{Introducción}

El presente texto plantea una reflexión sobre dos instituciones jurídicas relativamente nuevas: la regulación y el compliance. Ellas tienen una importancia determinante en el derecho de los negocios en la actualidad. El documento presenta una revisión de la principal literatura internacional sobre el asunto, veremos que la doctrina viene remozando algunos de los debates teóricos que han forjado tradicionalmente los análisis del derecho comercial. Se trata de nociones jurídicas que invitan a reexaminar el asunto de los derechos y las obligaciones de los sujetos jurídicos que desarrollan las actividades económicas consideradas por el derecho. En efecto, las figuras que vamos a analizar someten el ejercicio de la libertad de comercio y la libre iniciativa económica a condiciones legales peculiares. De esa manera, generan novedosas manifestaciones de problemas antiguos. Sin embargo, pese a su novedad, la discusión se estructura en torno de asuntos sobre los que ha gravitado históricamente el estudio de esa disciplina.

La regulación jurídica de la economía y el compliance son instituciones jurídicas polisémicas y complejas. Ello se debe, de una parte, a su origen norteamericano, hecho que plantea dificultades para su adopción en países de la tradición jurídica del derecho continental, como Colombia y Francia. El factor lingüístico genera confusión, pues la traducción simple de esos términos puede conducir a darles un sentido diferente y en ocasiones contrario a su contenido jurídico real. De allí que, en Francia, la profesora Frison-Roche (2016), quien es el principal referente académico en la materia, se ha rehusado a traducir el compliance como el derecho del cumplimiento o de la conformidad con la ley. En lugar de eso, ha preferido afirmar que esa es una expresión intraducible al francés. Algo similar ha ocurrido en el caso de la noción de regulación. Existe un consenso en la doctrina francesa, que ha alertado sobre un error en el que hemos incurrido en algunos casos en Colombia, al interpretar que ese término se traduce por la noción de reglamentación. En realidad, se trata de dos formas antagónicas de intervención del Estado en la economía (Sanclemente-Arciniegas, 2017).

De otra parte, la determinación del contenido de esas nociones presenta dificultades, ya que son usadas en variados ámbitos y con sentidos 
diversos. En algunos casos, ellas tienden a confundirse con la definición misma de derecho, hecho que las privaría de toda pertinencia. El alcance de la noción de regulación es analizado de manera detallada en la Sentencia C-150 de 2003 de la Corte Constitucional. En dicha providencia, la jurisprudencia expresa unas concepciones que dan cuenta de la complejidad de la institución y que se aproximan a la posición que en Francia ha sostenido la profesora Frison-Roche (1998, 2001, 2004). En efecto, la Corte advierte sobre las distintas definiciones legales de esa expresión, partiendo de una concepción extensa a otra más precisa. Sobre la acepción más amplia, la Corte afirma que "todas las decisiones del Estado respecto del funcionamiento de la economía y la organización de la sociedad constituyen una forma de regulación económica". No obstante, a renglón seguido, circunscribe la noción a la intervención estatal de naturaleza técnica y jurídica, en sectores económicos específicos. Dentro de ellos, el sistema jurídico tiende a expresar la voluntad política del Estado, dirigida a orientar el mercado hacia fines diferentes de los que se desprenden de la lógica mercantil.

El compliance es una noción más reciente que se desarrolla dentro de la misma dinámica de la primera institución. Sin embargo, la regulación se inscribe dentro de una corriente de sospecha frente a los políticos, a partir de la teoría de la captura del regulador, planteada por el Premio Nobel de Economía George Stigler (1971). Él alertó sobre la posibilidad de que los responsables políticos actúen bajo la influencia de los empresarios que financian sus campañas políticas. Por el contrario, el compliance expresa una cierta desconfianza hacia las empresas (Gonzalo, San-Jose E Ruiz-Roqueñi, 2017). En efecto, el grave incumplimiento de la ley por parte de las compañías ha llevado a los Estados a incrementar las exigencias normativas tendientes a asegurar que estas tomen conciencia sobre los riesgos que afrontan. La idea es obligarlas a que adapten su comportamiento, sus normas y su estructura organizativa, a fin de que eviten vulnerar valores jurídicamente relevantes. En tal sentido, autores como Teubner (2011) sostienen que los preceptos del compliance se han desarrollado bajo el impulso de grandes escándalos empresariales, como el de Enron o Siemens. En Colombia, el rol que ha jugado el denominado controler en el caso Odebrecht pone de manifiesto la pertinencia de planteamientos como los de Cortina (2000), según los cuales es 
necesario que las empresas emblemáticas refuercen la vigilancia sobre su propia conducta.

Para analizar los efectos de la regulación y del compliance en el ámbito de los derechos y las obligaciones de los sujetos económicos, dividiremos nuestra reflexión en dos partes. En la primera de ellas, observaremos que el advenimiento de un mercado regulado envuelve un control de acceso, hecho que limita derechos como el de la libre iniciativa económica y la libertad de comercio. Dicha limitación se expresa no solo al ingresar al mercado, sino que las normas del compliance tienden a prescribir la forma que debe tomar el comportamiento de los actores. En la segunda parte, veremos que las dos instituciones analizadas tienen manifestaciones que representan nuevas obligaciones para los actores económicos. La noción de regulación implica que los operadores deben incorporar fines extraeconómicos, impuestos por el derecho. Por su parte, la noción del compliance comporta compromisos cuyo incumplimiento puede dar lugar a sanciones severas, incluso de naturaleza penal. Con el fin de garantizar la obediencia de las nuevas obligaciones, el ordenamiento jurídico hace uso de nuevos mecanismos de sanción.

\section{Del control al acceso al control del comportamiento en el mercado}

El derecho comercial se ha fundado históricamente sobre la libertad económica. Esa potestad tiene como pilar esencial el derecho de acceder al mercado, que es el espacio en el que se concretizan las acciones que expresan esa libertad, como la producción, la oferta, la compra o la venta de bienes y servicios. La noción de regulación económica implica una mutación importante de los supuestos bajo los cuales el sistema económico permitía el ejercicio de esas libertades. Ella implica, de una parte, la renuncia del Estado a actuar directamente como protagonista en el mercado (Majone, 1997). De otra parte, ella envuelve la voluntad de un Estado modesto (Crozier, 2014), pero que pretende ser portador y garante de valores que no son atendidos por la lógica mercantil (Frison-Roche, 2004). De esa manera, la institución se sitúa en una doble oposición, por un lado, expresa una aspiración libertaria; 
y, por el otro, manifiesta el control estatal para el acceso al mercado. La forma en la que se desatan esas tensiones determina el ámbito real para el ejercicio de la libertad económica.

Una vez dentro del mercado, los actores del juego económico deben interiorizar que esa es una institución constituida legalmente. En ese sentido, el compliance profundiza la tendencia a responsabilizar a las empresas de su comportamiento. La idea es lograr que ellas interioricen restricciones legales destinadas a proteger intereses generales. El respeto voluntario de esas obligaciones no pasaría de ser una quimera. Por ello, instituciones como el compliance hacen uso de la coerción, incluso penal, con el fin de disciplinar comportamientos desviados. No obstante, el asunto plantea dificultades, pues el respeto de la libre iniciativa económica implica reconocer un ámbito para la autonomía para los operadores. En tal sentido, asistimos a la normalización de la vida económica (Turinetti, 2018). Ese fenómeno se expresa por la existencia de múltiples normas que trazan a los operadores económicos los estándares seguros por seguir, a fin de preservar valores jurídicamente relevantes. Así, el derecho opera, a la vez, como la mano amiga que guía y como el juez severo que constata las actuaciones no conformes (Thibierge, 2008).

\section{Control de acceso al mercado y libertad económica}

El acceso a los mercados ha sido desde la antigüedad una reivindicación esencial de los sujetos económicos. Ese es el ámbito natural para la realización de la libertad económica. En consecuencia, el acceso o la exclusión de ese espacio es un factor que determina tanto la posible creación de riqueza como la existencia misma como emprendedores. La importancia que ha tenido tradicionalmente el asunto se ha incrementado en los últimos años por el carácter esencialmente digital que ha tomado ese sitio. Al punto de que autores como Rifkin (2001) han denominado nuestra época como la edad del acceso. Una evolución caracterizada por un capitalismo en el que la posibilidad de acceder jugará un rol aún más preponderante que la institución de la propiedad privada, debido al predominio de redes que solo permitirán actuar a las personas conectadas. De allí que la caracterización de los diferentes 
tipos de Estados y de ordenamiento jurídicos tiende a fundarse en la posición que adopten ante este nuevo valor. En tal sentido, la figura del Estado regulador (Majone, 1996; Chevallier, 2004; Alviar \& Lamprea, 2015) expresa posiciones ambiguas en esa materia.

En un primer momento, en los países de la tradición romano-germánica, el advenimiento de la regulación económica se fundamentó en la necesidad de estructurar mercados libres y competitivos. Una mutación propia de la ideología neoliberal que impuso el abandono del Estado providencia (Majone, 1997). Por ello, la noción fue definida como una técnica de pasaje en mercados que se encontraban sometidos a un monopolio estatal y que iban a ser abiertos a la libre competencia (Frison-Roche, 1998). Esto se expresó en el retiro de las empresas estatales que operaban en condición de monopolio a fin de permitir el acceso de operadores privados. En ciertos casos, con el propósito de implementar una competencia económica, se subdividieron actividades que eran realizadas por un operador histórico monopólico. Por ejemplo, en el caso del mercado eléctrico, se dividieron las operaciones de generación, transporte y distribución. El objetivo era reducir el ámbito de las actividades monopólicas a aquellas efectivamente relacionadas con facilidades esenciales, e imponer la competencia, en aquellos sectores en los que las condiciones del mercado lo hacían posible (Millán, 2006).

Sin embargo, la relación de la noción de regulación con la libertad económica es problemática. Prueba de ello es que, en la tradición anglosajona, que es la fuente de la institución, la teoría de la regulación, tal y como fue formulada por Stigler, rechaza la regulación por ser una forma de limitar esa libertad. Así, la existencia de un mercado regulado implica normalmente una excepción al principio de libertad económica, como el previsto en el artículo 333 de la Constitución Nacional. En efecto, una de las funciones principales del regulador es la de autorizar el acceso al mercado respectivo. En consecuencia, esas instituciones se encuentran facultadas para verificar el cumplimiento de requisitos especiales, previa la autorización de acceso de los operadores al mercado. Esos requisitos operan como una barrera que impide ejercer libremente la actividad económica. Acceder al mercado, y ejercer la actividad económica, sin contar con la autorización previa de la autoridad de regulación 
puede generar sanciones. En algunos casos, como en el del ejercicio de la actividad financiera, ese hecho puede constituir un delito, según las previsiones del artículo 316 del Código Penal.

La existencia de autorizaciones administrativas que constituyen un control previo al acceso al mercado se encuentra naturalmente en tensión con los derechos a la libertad económica y a la libre iniciativa privada consagrados en la Constitución. Esos conflictos han sido objeto de pronunciamientos jurisprudenciales que generalmente han apoyado la facultad del Estado de imponer condiciones al ejercicio de esos derechos. Esa posición jurisprudencial parece sólida si se le mira desde la perspectiva del Estado como protector del interés general. No obstante, merecería un análisis detallado si se le considera a la luz de la tesis de Stigler, según la cual las autoridades de regulación se encuentran capturadas por los operadores económicos que actúan en el sector. En dicho caso, las autorizaciones administrativas previas pueden ser interpretadas como una barrera de acceso al mercado, que se erige en beneficio de las empresas que ya tienen presencia en el sector económico y en detrimento de las personas interesadas en acceder. De esa forma, la regulación económica, cuando el regulador se encuentra en situación de captura, se convierte en una amenaza para la libertad de los agentes que no cuentan con influencia ante las autoridades de regulación.

La importancia del asunto se ha incrementado conforme se ha expandido el ámbito económico sometido a la exigencia de autorización previa al ingreso. En tal sentido, la Corte Constitucional, en la Sentencia C-1072 de 2002, ha observado que, en el Estado social de derecho, el ámbito de esas autorizaciones administrativas se ha visto ampliado de manera extraordinaria. Ya no opera exclusivamente a partir de la noción de orden público, concebido en la triple dimensión de tranquilidad, seguridad y salubridad, sino que se ha extendido al campo de la actividad económica, dentro del cual adquiere una nueva dimensión en función del papel que al Estado le corresponde en la dirección de la economía y la promoción del interés general. En esa misma providencia, se afirma que la autorización administrativa comporta la existencia de una prohibición previa sobre actividades económicas de los particulares. De modo que es razonable plantear que los principios de libre iniciativa y libertad económica hoy operan más como la excepción que como la regla. 
Es conveniente anotar que la generalización de mercados regulados conduce a vastos sectores de la población al ejercicio de la actividad económica informal. Esto como producto de las dificultades para cumplir las condiciones que exigen las autorizaciones previas. Esas condiciones se transforman en obstáculos que impiden el ejercicio de los derechos a la libertad económica y a la libre iniciativa privada. Así, la imposibilidad de acceder al mercado tiene efectos que van más allá del ámbito económico. Frente a ello, el ejercicio de esas libertades exhibe en este aspecto su carácter de derecho fundamental. En efecto, el respecto de la dignidad humana de sectores vulnerables de la sociedad implica permitirles ejercer una actividad económica en los términos establecidos en la ley. En ese sentido, estudios como los de Chambers, McLaughlin y Stanley (2019) han encontrado que las barreras para acceder al mercado afectan especialmente a personas de bajos ingresos. De esa manera, se incrementan los niveles de desigualdad social, ya que esos sectores se ven condenados a realizar la actividad económica de forma ilegal, obteniendo menores beneficios y asumiendo costos adicionales derivados de ese hecho.

En el mismo sentido, análisis como los de North et al. (2007) han concluido que las barreras para acceder al mercado son un problema propio de los países en desarrollo. Ese estudio concluye que, en las economías de los Estados más ricos, las autorizaciones administrativas para el acceso al mercado son menores. Por tal razón, el resultado del juego económico es determinado por la competencia. Por el contrario, en los países pobres los obstáculos son múltiples y su funcionamiento es afectado por las influencias en busca de rentas. Lo anterior se relaciona estrechamente con la adopción, por parte de los primeros países, de instituciones de regulación independientes del poder político. En las economías (como la colombiana) en las que los políticos controlan las autoridades de regulación, nombrando y removiendo libremente a directores o imponiéndoles sus decisiones, los resultados de la interacción económica no dependen solo de las calidades competitivas de las empresas. El éxito o el fracaso del proyecto económico son determinados por las influencias que ellas puedan ejercer ante las instancias políticas. Para describir ese fenómeno, la doctrina internacional ha acuñado el término de crony capitalism (Aligica \& Tarko, 2014). La expresión hace 
referencia a las economías en las que las decisiones de las entidades de regulación tienden a beneficiar a los actores económicos que cuentan con influencia política. Esos son beneficios que obtienen de manera indebida sectores minoritarios, en detrimento de las mayorías que carecen de acceso a las esferas de decisión.

Por ello, en el contexto de un Estado social de derecho, la existencia de numerosos ciudadanos excluidos del acceso al mercado y condenados a la economía informal plantea cuestionamientos. Esa situación incita a repensar el papel que deben jugar tanto las instituciones de regulación económica como aquellas encargadas de garantizar la efectividad de los principios y derechos consagrados en la Constitución. En efecto, la norma constitucional (art. $2^{\circ}$ ) ordena a las entidades públicas comprometerse con la efectividad de los derechos a la libertad económica y a la libre iniciativa privada consagrados en la Carta. En esa perspectiva, resulta pertinente la observación que realiza Foucault (2007) al analizar el ordoliberalismo alemán. Dentro de ese marco conceptual, íntimamente ligado con la economía social de mercado, se ha entendido que, en un modelo económico fundado sobre la libertad económica, la actuación de las autoridades debe dirigirse a generar y proteger activamente el principio en torno del cual se articula el sistema: la libertad para todos.

Analizado en esa perspectiva, el rol de las autoridades de regulación no debería ser solo el de controlar el acceso al sector. Ellas deberían incitar y facilitar el emprendimiento de nuevos operadores, estimulando la libre competencia y protegiendo la libertad económica y la libre iniciativa de agentes que no están presentes en el mercado. En tal sentido, debe tenerse en cuenta que acceder formalmente al mercado, entendido como los datos, las redes o el espacio público, puede representar desafíos insuperables para amplias capas de la población. En consecuencia, el compromiso de las instituciones estatales con la efectividad de los derechos a la libertad y la libre iniciativa económica debería concebirse en los términos de libertad como capacidad a los que hace referencia Amartya Sen (Cejudo, 2007). Así, el compromiso del Estado con la efectividad de esos derechos implica una especie de asociación con los ciudadanos interesados en ejercerlos. En ese sentido, la actividad del Estado debería orientarse a potenciar el emprendimiento de los ciudadanos. Para ello, antes que la función de control de acceso, 
se debería privilegiar la de generar mercados accesibles, abriendo los espacios económicos a sectores tradicionalmente excluidos.

\section{Control del comportamiento en el mercado y libre iniciativa económica}

La consagración constitucional de la libre iniciativa privada (art. 333) recoge una tradición dentro la cual el ámbito de ejercicio de esa libertad tiene como límite natural el bien común. A fin de proteger ese derecho económico, sin afectar otros bienes y valores constitucionales, se ha apelado habitualmente a la responsabilidad social de las empresas y a las actividades estatales de regulación, vigilancia y control (Sentencia C-228/10). Sin embargo, en la actualidad, esos criterios tradicionales son cuestionados por nuevas instituciones jurídicas, como el derecho del compliance. Dentro del marco propio de esa concepción, la responsabilidad social de las empresas deja de ser un asunto puramente voluntario, asociado con operaciones de marketing. De allí que las actividades de regulación, vigilancia y control tienden a desplazarse de las esferas públicas hacia las instancias privadas de las empresas. De esa manera, el ámbito de la libertad económica queda sometido a diferentes tipos de normas, que no tienen su origen exclusivo en la función reguladora del Estado.

En efecto, la noción compliance representa una ruptura con categorías tradicionales del derecho de los negocios, por ello, los doctrinantes hacen referencia a un nuevo mundo (Gaudemet, 2016), una nueva era (Arroyo \& Nieto, 2013), una nueva cultura (Cordier-Palasse, 2016) o un antes y un después (Frison-Roche, 2018). No obstante, la complejidad del compliance radica no tanto en su novedad, sino en intentar fundir en una sola institución influencias provenientes de diferentes lógicas (liberalismo-estatismo) y categorías jurídicas (derecho público-derecho privado) y actores. Allí se entrelazan, de una manera novedosa, las tensiones habituales entre el ejercicio de la libertad económica y los límites asociados a la protección del interés general. En ese contexto, el Estado cede modestamente tanto la iniciativa legislativa como la aspiración a proteger por su acción directa valores jurídicamente relevantes. En lugar de ello, las empresas son investidas de competencias de protección del interés general que, en principio, correspondería desarrollar al Estado. 
Para alcanzar esas misiones, los agentes privados son impulsados a utilizar herramientas que, en tiempos pasados, eran concebidas como prerrogativas de las entidades públicas: la expedición de normas obligatorias (Teubner, 2011).

Así, en el derecho del compliance, el comportamiento de las empresas en el mercado es objeto de control por normatividades de tres orígenes diferentes: estatal, empresarial y técnico. En cuanto a las normas estatales, la intención legislativa se orienta a realizar el primer paso dentro del proceso de gestión del riesgo: la identificación de las vulnerabilidades que debe afrontar el desarrollo del objeto social de la empresa. Ese enfoque jurídico, basado en los principios de prevención y precaución, se manifiesta como un instrumento que propone nuevas formas en las que las compañías deben comportarse en el mercado y gobernarse (Charpentier, 2014). En efecto, existe un conjunto diverso de normas estatales que identifican riesgos en distintos ámbitos de la actividad económica. Dentro de cada uno de ellos, la noción de compliance no se limita a obligar a que las empresas cumplan las normas a las que se encuentran sometidas, sino que pretende además controlar el comportamiento de los operadores, proporcionando a los administradores los estándares que deben seguir para el manejo de estos. Así, de acuerdo con el objeto de la empresa, el derecho le identifica los riesgos a los que se encuentra expuesta. De modo que el administrador diligente deberá, en primer lugar, atender las disposiciones legales que lo orientan en la identificación del tipo de riesgos que debe afrontar de manera prioritaria y en la forma de gestionarlos.

Las normas estatales en materia de compliance se refieren a asuntos muy diversos, como riesgos ambientales, sanitarios, o de manejo de datos personales. Sin embargo, se destacan las disposiciones que recogen compromisos internacionales de los Estados. Tal es el caso de las normas que exigen que las empresas adopten un sistema para la prevención del lavado de activos y la financiación del terrorismo (Sarlaf). Tanto la decisión de adoptar esas normas como su contenido y los criterios fijados para su seguimiento responden a iniciativas internacionales lideradas por el GAFI (Fitzgerald, 2004). Algo similar ocurre con las normas destinadas a establecer la responsabilidad de las personas jurídicas por actos de corrupción. Así, en Colombia, la Ley 1778 de 2016 desarrolla las 
disposiciones de la Convención de las Naciones Unidas contra la Corrupción, como el artículo 12, según el cual los Estados deben vincular al sector privado a la lucha contra ese flagelo, promoviendo la formulación de normas y procedimientos encaminados a salvaguardar la integridad de las entidades privadas pertinentes. Para ello, la Convención establece que se deben adoptar sanciones para prevenir la corrupción en el sector privado y, cuando sea procedente, establecer sanciones civiles, administrativas o penales eficaces, proporcionadas y disuasivas en caso de incumplimiento de esas medidas.

En cuanto a las normas que tienen su origen en las empresas, el poder normativo de los códigos de conducta privados es una institución jurídica propia del derecho del compliance que ha ganado el interés de la doctrina desde los años ochenta. Acerca de su importancia reflexionó en forma pionera el gran jurista francés Gerard Farjat (Sanclemente-Arciniegas, 2018), él fue uno de los primeros en vislumbrar la magnitud de los efectos económicos y políticos que pueden derivarse de la iniciativa normativa privada. En efecto, el poder normativo de las empresas, especialmente las multinacionales, se agrega a su poderío económico. Debido a ello, los estándares normativos adoptados por esas compañías pueden tener impactos superiores a las normas estatales. Esas disposiciones logran afectar a numerosas personas, como trabajadores, proveeros, contratistas o consumidores, en diferentes países del mundo; ellas se erigen como modelos para jueces, legisladores o entidades de regulación. Su desarrollo se inscribe dentro de la mutación del derecho a la que ha hecho referencia Santos (1987), quien ha sostenido que la aparición de diversas fuentes de normas sería una manifestación propia de la posmodernidad. En ella, el monopolio normativo del Estado desaparece, dando lugar a un pluralismo que implica la coexistencia de varios sistemas jurídicos, los cuales circulan por diferentes redes, generando relaciones cada vez más complejas entre el derecho, la economía y la sociedad.

Las reflexiones de Farjat han sido profundizadas en los últimos años por Gunter Teubner (2011), quien ha señalado que los códigos de conducta de las grandes empresas privadas son constituciones en el sentido propio del término. En esa misma perspectiva, asevera que esos cuerpos normativos contienen normas de diferentes jerarquías, que regulan conflictos entre ellas, y de esa manera condicionan la producción 
normativa de la organización. En el nivel superior, ubica los principios de la constitución corporativa, que señalan los valores que guían la empresa. En el nivel medio, están las normas de aplicación y monitoreo de las disposiciones superiores, mientras que el nivel más bajo incluye disposiciones que establecen instrucciones concretas de conducta en aspectos diversos. La fuerza normativa que irradia esos preceptos puede tener un alcance mundial, afectando distintos tipos de sujetos, dentro de los cuales se destacan las empresas de menor tamaño. Así, las normas de compliance adoptadas por una firma grande tienen como efecto influenciar el comportamiento en el mercado de otras compañías (Nielsen E Parker, 2008). La idea es evitar que la realización de los valores y principios de una empresa se vea frustrado por el contagio de prácticas de compañías con las que se establecen relaciones estrechas.

En cuanto a la influencia de las normas técnicas sobre el comportamiento en el mercado, conviene recordar que Michel Foucault (1976) había observado que, en nuestros tiempos, los nuevos dispositivos del poder no se asocian tanto con el derecho, sino más bien con la técnica. De allí se puede explicar la abundante cantidad de normas técnicas que proporcionan a las empresas los estándares de actuación en el mercado. En efecto, el compliance puede ser entendido como un proceso de control de riesgos normativos determinado por la experticia técnica. Por ello, las iniciativas normativas de instituciones como la Organización Internacional de Normalización (ISO) tiende a superar las decisiones estatales en la materia. Esa entidad ha emitido un conjunto de normas técnicas internacionales relativas al asunto, iniciando por la norma ISO 19600, que establece guías para la implementación de los sistemas de compliance; y la norma ISO 31000, que prevé directrices técnicas para la gestión del riesgo en general. Además, ha adoptado criterios técnicos para la gestión de la responsabilidad social de las empresas (ISO 26000), para el manejo de riesgos ambientales (ISO 14000), para las compras sostenibles (ISO 20400) y para la gestión de riesgos de soborno y ética empresarial (ISO 37001), entre otras.

Así las cosas, la multiplicación de normas técnicas junto con las disposiciones estatales y los códigos de conducta de las otras empresas operan como nuevos controles normativos al comportamiento de los operadores en el mercado. Podría pensarse que de esa manera se limita 
drásticamente la libre iniciativa económica. No obstante, debe tenerse en cuenta que esos dispositivos normativos han florecido en una época en la que se valoran de forma más positiva las iniciativas económicas privadas que las públicas. Esto implica el advenimiento de un marco jurídico complejo, al que debe adaptarse la autonomía de los agentes económicos. Dicho marco no afecta de manera idéntica a todos los actores; como hemos visto, las grandes compañías pueden, gracias a su poder normativo, imponerles sus normas a las pequeñas. En tal sentido, conviene resaltar que el tamaño de las empresas es un aspecto relevante para determinar el tipo de obligaciones que estas deben soportar. De lo contrario, el cumplimiento de las normas de compliance puede convertirse en un nuevo factor, que excluye a los pequeños operadores en beneficio de los grandes conglomerados.

\section{Del objetivo de ganar dinero a la obligación de proteger el interés general}

El 13 de septiembre de 1970 Milton Friedman publicó en el New York Times Magazine un famoso artículo en el que sostuvo que la única responsabilidad de las empresas es generar ganancias para sus propietarios. En su concepto, dado que la sociedad es una ficción jurídica, no es institución apta para adquirir obligaciones de ese tipo, esa responsabilidad sería predicable exclusivamente de las personas naturales que gobiernan las empresas. Pues bien, el derecho de la regulación y el del compliance configuran desarrollos legislativos diametralmente opuestos a las proposiciones de Friedman. Estas nuevas instituciones confrontan los dos argumentos que ese autor expone: de una parte, tienden a imponer a las compañías obligaciones diferentes a la mera generación de ganancias; de la otra, sancionan directamente a la empresa por las acciones que vulneran bienes protegidos jurídicamente. Así, el compliance se fundamenta en la responsabilidad, administrativa y penal de las personas jurídicas, como una figura diferente de la responsabilidad de sus administradores.

Por su parte, el derecho de la regulación económica tiene como objetivo esencial garantizar que los operadores que actúan en el mercado 
integren en su accionar valores que no se desprenden de la lógica mercantil. En efecto, pese a que las dos instituciones que aquí se analizan son propias de una concepción en la que el mercado juega un rol preponderante, ellas son las herramientas legales a través de las cuales el Estado condiciona el funcionamiento del sistema económico. Así, la regulación no se dirige a permitir que el mercado funcione normalmente, sino a asegurarse de que en su dinámica no vulnere valores superiores, como la dignidad de las personas. Se trata, una vez más, de una construcción compleja en la que las empresas, actores privados por excelencia, son llamadas a proteger activamente, además de los intereses propios de sus accionistas, fines monumentales (Frison-Roche, 2016) asociados con la prevalencia del interés general.

\section{Nuevas obligaciones de las empresas}

Tanto el derecho de la regulación como el del compliance implican el advenimiento de nuevas obligaciones para las empresas. Ese fenómeno se explica por los efectos de dos fenómenos que operan de manera simultánea. En primer lugar, el nuevo contenido obligacional es consecuencia de la expansión de la presencia de operadores privados hacia sectores que tradicionalmente se situaban por fuera de la lógica mercantil. Como hemos señalado, la regulación jurídica se asocia con el retiro del Estado como actor principal en el mercado, especialmente en el sector de los servicios públicos. En la medida en que el Estado desaparece, el mercado conquista nuevos ámbitos de la actividad humana; por ello, el derecho debe seguir la expansión de la dinámica mercantil con el fin de garantizar que su funcionamiento respete valores superiores. Así, al ocupar el lugar que antes correspondía al Estado, los operadores privados se ven forzados a asumir obligaciones que se derivan de esa condición.

En tal sentido, los operadores privados que asumieron el rol de las empresas estatales que prestaban servicios públicos han sido obligados a cumplir nuevas obligaciones derivadas de la naturaleza de la actividad que desarrollan. Tal es el caso del respeto de valores como la universalidad, la continuidad del servicio o la no discriminación. En todos esos casos, el marco normativo que permitió el ingreso del operador privado impuso el respeto de ese tipo de obligaciones. Esto bajo el entendido 
de que esos principios protegen bienes meritorios que todo ser humano requiere para su subsistencia. Naturalmente, esto no implica que las empresas privadas destinen parte de su patrimonio a cubrir ese tipo de bienes, sino que, dentro de la estructuración de los nuevos sectores económicos, se concibió la forma de financiar adecuadamente esas prestaciones. Para ello se utilizaron herramientas que van desde los subsidios cruzados hasta la autorización de acceder a mercados atractivos bajo la condición de llevar el servicio a zonas apartadas.

En segundo lugar, el desplazamiento de las actividades de regulación, vigilancia y control de las esferas estatales hacia el ámbito interno de las compañías ha implicado la aparición de nuevas obligaciones para las empresas. La primera de ellas es precisamente la de tomar conciencia de ese hecho y, en consecuencia, adoptar las instituciones necesarias para garantizar que se lleven a cabo, con sus instrumentos internos, esas labores de vigilancia. En esa perspectiva, los códigos de ética aparecen como el principal instrumento a través del cual las empresas expresan su voluntad de cumplir las normas a las que se encuentran sometidas. Además, esos códigos han sido definidos (Martín, 2000) como un pacto ético que asegura la cohesión moral de empresa, en una lógica que da prevalencia al interés social de la compañía sobre los intereses personales de los individuos que la integran. Su implementación facilita que la organización alcance objetivos diversos, como cumplir sus obligaciones legales, estructurar una cultura empresarial propia, gestionar eficazmente los recursos humanos o legitimar los roles gerenciales. Sin embargo, dentro de los fines que ellos persiguen se han destacados aquellos que los identifican como la base de actividad del compliance (Rodríguez, 2015).

En Colombia, los desarrollos del compliance han sido muy escasos comparados con los avances que a ese respecto han hechos otros países. Pese a ello, el artículo 23 de la Ley 1778 de 2016 facultó a la Superintendencia de Sociedades para determinar qué personas jurídicas deben dotarse de programas de ética empresarial, teniendo en cuenta aspectos como el monto de sus activos, sus ingresos, el número de empleados y el objeto social. En el mismo sentido, el artículo $7^{\circ}$ de la misma ley identifica como criterios de graduación de las sanciones previstas en ella la existencia, ejecución y efectividad de programas de transparencia y ética empresarial o de mecanismos anticorrupción dentro de la empresa. 
En el derecho de países como Francia (Schiller, 2017; Lequet, 2017), al conjunto de nuevas actividades que la empresa debe desarrollar en aplicación de las normas de compliance se le ha denominado como la obligación de vigilancia; en la legislación española, se ha hecho referencia al deber de control (Gómez-Aller, 2013). En todos los casos, se hace referencia a una obligación a cargo de los agentes económicos de tomar iniciativa en forma proactiva con miras a vigilar su comportamiento, evitando incurrir en actividades que vulneren bienes protegidos jurídicamente y los expongan a sanciones administrativas o penales.

De esa manera, las empresas no solo son forzadas a interiorizar valores de los que es portador el ordenamiento jurídico. Además, han sido compelidas a adoptar posiciones éticas frente a principios que, en algunos casos, se relacionan estrechamente con el desarrollo de su objeto social, pero que, en otros, se refieren a preocupaciones sociales en general. De allí que, en la actualidad, las empresas aparecen como fuentes normativas en asuntos tan variados como la protección del medio ambiente, la lucha contra la corrupción o la salvaguarda de los derechos humanos. En tal sentido, autores como Carlos Requena (2018) señalan la importancia que tienen las herramientas del derecho del compliance en un nuevo rol que debe ser asumido por las empresas: la protección de los derechos humanos. Las herramientas de compliance dotan las compañías de instrumentos que les permiten actuar como protagonistas en el sistema mundial de protección de los derechos humanos. Así, la protección de los datos personales, la lucha contra la corrupción y, en general, la protección de la dignidad de la persona humana han dejado de ser concebidos como una obligación exclusiva de las autoridades públicas.

A fin de cumplir con ese tipo de compromisos, las empresas vienen adoptando nuevos estándares normativos. Así, en análisis como el de Corinne Gendron se constata que desde el año 2006 se ha producido una aceleración de una tendencia global a la adopción de instrumentos normativos de origen empresarial. En dicho estudio se afirma que, para esa época, en Estados Unidos entre el 80\% y el $93 \%$ de las grandes empresas tienen un código o una carta ética, y este porcentaje llegaría al 77\% en Japón y al 62\% en Francia. Conviene tener en cuenta que los operadores económicos son forzados a adoptar ese tipo de códigos no solo por la fuerza normativa de las normas estatales, sino por las 
presiones que se desprenden del mercado. En efecto, el compliance implica una toma de conciencia de la relevancia material de la reputación, tal y como ha sido planteado en los estudios de Eccles, Newquist y Schatz (2007). En ese análisis se concluye que el prestigio de la compañía tiene efectos que van más allá de aspectos exclusivos de imagen y toca aspectos que determinan el desempeño financiero.

Además de los programas de ética, merece destacarse la obligación de adoptar un compliance officer. Esta es una institución propia de los sistemas para la prevención del lavado de activos y la financiación del terrorismo (Sarlaf), dirigida inicialmente a las entidades que realizan actividades financieras, pero que ahora se ha extendido a las empresas del sector real. El compliance officer es la persona que se encuentra a cargo del liderazgo estratégico y operativo de ética corporativa (Weber \& Fortun 2005). En ese rol se encarga de la difusión de esas políticas, implementa los procedimientos internos de sanción en caso de incumplimiento, monitorea su obediencia y se asegura de la que la política deje de ser un simple documento e impregne realmente el quehacer cotidiano de la empresa. A fin de permitirle llevar a cabo esas misiones de manera adecuada, el programa de cumplimiento debe dotar al compliance officer de un estatuto interno que le garantice contar con independencia frente a la administración y con recursos humanos y financieros adecuados para implementarlo. Adicionalmente, debe garantizarle acceso directo a la alta dirección, de manera que los actos contrarios a la ética de los negocios puedan ser comunicados sin la interferencia del gerente.

De otra parte, los programas de ética deben consagrar normas que protejan a los lanzadores de alertas. Esa figura corresponde en inglés al término whistleblowing, que hace referencia a una manera en la que, en otros tiempos, los policías británicos alertaban sobre la comisión de delitos, haciendo sonar un pito. En los programas de compliance mediante esa figura se permite que cualquier persona que integre una compañía pueda alertar sobre la realización de actos ilícitos o sospechosos. La importancia de procedimientos que permitan denunciar de forma segura, anónima y confidencial ha sido juzgada como determinante para que las personas se animen a hacerlo. Se trata, de un lado, de estimular a las personas a que denuncien actos deshonestos; $y$, del otro, de establecer procedimientos claros que garanticen que quienes denuncien no sufrirán 
efectos negativos por ello. De allí que diferentes entidades internacionales han producidos cuerpos normativos completos en los que se regula de manera detallada esos procedimientos (Vandekerckhove \& Lewis, 2012). En consecuencia, el programa de ética puede integrar las guías que a ese respecto han redactado entidades como Transparencia Internacional, la Unión Europea o la Cámara de Comercio Internacional.

Así las cosas, el derecho del compliance confronta las tesis de autores como Friedman, pues no solo recoge la teoría de la responsabilidad empresarial, que ellos cuestionan, sino que la profundiza, dando lugar a un nuevo conjunto de obligaciones a cargo de la empresa. De allí que Tricot (2016) haga referencia a un fenómeno de juridificación de la responsabilidad social empresarial. Así, lo que bajo la perspectiva gerencial podía ser interpretado como una simple operación de marketing, ahora ha tomado un cariz coercitivo, propio de las normas de orden público que se imponen de forma imperativa a la empresa. Pese a ello, la tendencia no implica una vuelta al estatismo, sino que se trata de una expresión propia del derecho de la regulación, dentro del cual el Estado deja de actuar directamente en diferentes ámbitos económicos, cediendo su iniciativa a las empresas. Mediante el compliance lo que se viene cediendo es la actividad de regulación, control y vigilancia de los operadores económicos. No obstante, no se trata solo de la autorregulación, sino de una nueva obligación que debe ser ejercida por los agentes económicos y en beneficio de la sociedad. Por tal razón, Adán Nieto (2013) evoca una nueva privatización: la de asuntos como la lucha contra la corrupción.

\section{Nuevos mecanismos de sanción}

Las nociones de compliance y regulación fundan su carácter jurídico en la coerción. Ese factor distingue las obligaciones que ellas contienen de los compromisos asumidos dentro de la responsabilidad social empresarial o la autorregulación económica. En el interior del sistema jurídico, el ejercicio de la facultad sancionatoria para reprimir las infracciones propias de esas instituciones también presenta especificidades novedosas. En un primer momento, veremos que esa facultad tiende a escapar de las autoridades judiciales, que tradicionalmente son las llamadas a 
imponer sanciones. En su lugar, aparecen los reguladores, entidades de naturaleza administrativa, que son facultados para reprimir las actuaciones contrarias a derecho. En un segundo momento, observaremos que el ejercicio de la facultad sancionatoria es delegado a las empresas, como parte del desplazamiento de la facultad de regulación al que hemos hecho referencia anteriormente. En tercer lugar, destacaremos el uso de las sanciones de naturaleza penal, como una novedad propia del derecho del compliance.

En cuanto al primer aspecto, el traslado de la facultad sancionatoria de autoridades judiciales a instancias administrativas es un fenómeno que desafía el esquema clásico de la división de poderes (Epron, 2011; Strauss, 1984). En efecto, la naturaleza de las sanciones que pueden imponer las entidades de regulación desborda los límites propios de la facultad de sanción de la administración. Así, decisiones sobre la autorización del acceso o la expulsión del mercado conciernen, literalmente, a la vida o muerte de las personas jurídicas que han sido creadas precisamente con ese objeto, pero que la sanción hace imposible. De allí que internacionalmente se ha aceptado que, pese a ser autoridades administrativas, los reguladores son jueces en el sentido propio del término (Koering-Joulin, 1990). En consecuencia, con el fin de respetar el derecho fundamental al debido proceso de los operadores económicos, debe garantizarse la independencia y la imparcialidad de las entidades de regulación. En Colombia, tal independencia en realidad no existe, ya que las autoridades de regulación se encuentran sometidas a la influencia determinante del Presidente de la República.

La tendencia a transferir la competencia para imponer sanciones de los jueces a los reguladores se ha explicado por las dificultades de la jurisdicción para decidir asuntos de naturaleza económica. De allí que autores como Shleifer (2012) hablan del fracaso de los jueces y del advenimiento de los reguladores; según él, acceder a la jurisdicción implica costos importantes y los jueces deciden las controversias en forma parcializada e impredecible. Por ello, los reguladores aparecen como instancias más aptas para resolver de una manera segura, ágil y técnica los litigios económicos. Consideraciones similares han sido expresadas en un estudio del Senado francés mediante el cual se evaluó la tendencia a adoptar reguladores independientes (Gélard, 2006). Dicho análisis 
sostiene que esas nuevas instituciones cuestionan la capacidad de las autoridades judiciales para el cumplimiento efectivo de sus misiones tradicionales. En este sentido, se ha señalado que la transferencia de la facultad de sanción a los reguladores corresponde a una desconfianza ante el poder judicial, especialmente una evaluación negativa de la experticia técnica de la jurisdicción para comprender la complejidad de los asuntos traídos a su conocimiento.

El traslado de la facultad sancionatoria de entidades de naturaleza judicial a instituciones administrativas corresponde entonces a una tendencia internacional, que pretende mejorar la capacidad represiva del Estado. De esa manera se garantiza que las empresas cumplan las obligaciones a las que se encuentran sometidas, pues los reguladores constituyen mecanismos de acción pública más ágiles y eficientes. Sin embargo, la eficacia de tales instrumentos depende en gran medida de la independencia y la imparcialidad que se les reconozca. En Colombia, la competencia para sancionar no se encuentra aislada de la influencia política (Sanclemente-Arciniegas, 2019). En ese aspecto, la situación de la telefonía celular resulta ejemplar; de acuerdo con los estudios de la OCDE (2014), se trata de un sector sometido a la presencia dominante de un actor que presta servicios de mala calidad y a altos precios. Pese a ello, el artículo 63 de la Ley 1341 de 2019 otorga la facultad para sancionar a los operadores a un ente netamente político, como el ministerio que orienta el sector. En el mismo sentido, en el sistema financiero, se han hecho denuncias públicas (El Espectador, 2013; Taboada, 2012), según las cuales lobistas con influencia ante el presidente habrían logrado la remoción de un regulador que se oponía a prácticas inseguras de una entidad vigilada.

En cuanto al segundo aspecto, las facultades de sanción de las que goza la empresa evocan cambios fundamentales dentro del ordenamiento jurídico. En tal sentido, Teubner (2011) indica que en ese dominio se ha presentado una inversión del paradigma tradicional. Lo anterior debido a que, usualmente, se entendía que las normas de las empresas hacían parte del soft law y que las estatales constituían la expresión de la ley dura. No obstante, ese autor arguye que, debido al derecho del compliance, las normas estatales tienden a devenir meras recomendaciones, expresiones propias del soft law, mientras que las normas de las 
empresas devienen leyes duras, gracias al poder de sanción que ejercen las autoridades internas de compliance. De esa manera, la empresa puede desplegar una actividad eficaz tendiente a controlar su orden interno. Por ello, la competencia para reprimir se dirige esencialmente a garantizar el respeto de las disposiciones que ella misma ha adoptado. Esos preceptos son de forzoso cumplimiento para empleados, contratistas o proveedores, dado que se establecen como obligaciones dentro de los contratos que la empresa celebra con ellos.

La importancia de que la empresa ejerza el derecho de sanción radica en que, de esa manera, se protege a sí misma. En efecto, el despliegue de expresiones normativas y sanciones demostraría que la firma no es la responsable de las acciones reprensibles que cometen sus empleados, proveedores o contratistas. En tal sentido, Manacorda (2015) hace referencia a los programas de cumplimiento como constituciones de un orden jurídico interno de las empresas. Dentro de dicho orden se distinguen unas normas de carácter sustancial que establecen las conductas prohibidas y otras de naturaleza procesal que organizan los procedimientos internos a los que debe sujetarse la imposición de sanciones. En la perspectiva procesal, ese penalista destaca, además, la utilidad de ese tipo de programas dentro de los procesos penales en los que se cuestiona la conducta de la empresa. En tal caso, los programas de cumplimiento serían un elemento importante en la estrategia de defensa, puesto que demostrarían la ausencia de negligencia en el control de las actividades, hecho que conduciría a impedir la configuración de una responsabilidad penal.

Conviene resaltar que el poder disciplinario que ejerce la empresa sobre sus empleados es un asunto tradicional, que dentro de la noción de compliance adquiere ribetes especiales. De una parte, por cuanto no afecta exclusivamente a sujetos que tienen relaciones de naturaleza laboral con la compañía, sino que puede ser aplicado a contratistas, proveedores o usuarios. De la otra, por cuanto los intereses que mediante ese poder se protegen son diferentes. La actividad represiva que ejercen las empresas participa de una evolución de las concepciones de lo público y lo privado; en tal sentido, resulta pertinente el análisis del ejercicio del poder en derecho privado que realiza Gaillard (1985). Según ese autor, esa potestad puede ser concebida como una prerrogativa que otorga 
la ley a las empresas, para que sea ejercido en beneficio de un interés distinto al suyo. Así, el interés que protege la facultad sancionadora de la empresa no es solo el interés privado de esa persona jurídica, sino que se aparenta con el interés general, que preservan las instituciones de regulación.

En cuanto al tercer aspecto, el cumplimiento de las obligaciones propias del derecho de compliance ha adquirido una importancia fundamental debido a la instauración de una responsabilidad penal en cabeza de las empresas; esa es una novedad mayor propia de la nueva institución jurídica (Arroyo \& Nieto, 2013; Rotsch, 2012). Como hemos dicho, los desarrollos del compliance en el ordenamiento jurídico colombiano son muy escasos, sin embargo, el artículo 35 de Ley 1778 de 2016 establece la responsabilidad penal de las personas jurídicas independientemente de las responsabilidades penales individuales a que hubiere lugar. En tal sentido, la norma remite a sanciones como la suspensión y cancelación de la personería jurídica, el cierre temporal de los locales o establecimientos abiertos al público cuando existan motivos fundados que permitan inferir que se han dedicado total o parcialmente al desarrollo de actividades delictivas. En consecuencia, nos encontramos justo en el escenario que Friedman consideraba un imposible jurídico. Castigar penalmente a las personas jurídicas es una iniciativa que plantea dificultades y debates en la dogmática penal, pero hoy constituye una tendencia mundial ante la expansión de la criminalidad empresarial.

La represión de esa criminalidad se asocia con la noción de empresa como la actividad económica organizada de la que habla el artículo 25 del Código de Comercio. Así, desde los primeros análisis que se realizaron sobre el asunto, se consideró que los elementos dolosos, propios de la responsabilidad penal de las personas naturales, deberían ser sustituidos por la apreciación acerca de la forma en la que la empresa se organiza para hacer frente a los riesgos que el derecho le pone de presente (Stone, 1980). Por ello, la observancia de obligaciones como respetar la independencia del compliance officer, adoptar un código de conducta o un programa de cumplimiento son asuntos relevantes al momento de determinar si una empresa es penalmente responsable. La presencia de ese tipo de instituciones demostraría que la empresa ha asumido seriamente la obligación de interiorizar las funciones de 
regulación, control y vigilancia. Por el contrario, la ausencia de medidas organizativas adecuadas puede ser considerada como un indicio de esa responsabilidad. En tal sentido, Missas (2018) señala que la noción de culpabilidad penal de las personas jurídicas se fundaría en la idea de un desorden organizacional, que se encuentra al origen de la vulneración de bienes protegidos jurídicamente.

\section{Conclusiones}

La regulación y el compliance son nuevas instituciones jurídicas que remozan viejas cuestiones acerca de los derechos y las obligaciones de los operadores económicos. Ellas interpelan también el rol del derecho y del Estado, planteándoles novedosos desafíos para la protección de la libertad económica y la libre iniciativa privada, al tiempo que salvaguardan el interés general. La manera en la que se resuelven esos retos dista de ser evidente, pues se trata de asuntos que no se adaptan a paradigmas tradicionales, sino que mezclan de forma inusitada principios originados en diferentes lógicas. Así, la libre empresa deja de ser concebida como un privilegio de los poderes económicos, para convertirse en un derecho reivindicado por clases populares excluidas del mercado formal. En el mismo sentido, la defensa de valores protegidos jurídicamente deja de ser una competencia exclusiva de las instituciones públicas, para convertirse en una obligación de los agentes económicos. La complejidad del asunto impone la adopción de una visión pragmática, que guíe en la implementación del modelo de economía social de mercado propuesto por la Constitución.

En ese empeño, resulta necesario que el Estado colombiano, de un lado, concluya el proceso modernizador que planteó el constituyente de 1991, separando de forma clara el ejercicio del poder político y las funciones que ejercen las entidades de regulación económica. De ese modo, se garantizan los derechos de todos los que aspiran a acceder a mercados abiertos, en especial de quienes, pese a tener la voluntad de emprender, no cuenten con influencias políticas. En la misma forma, la observación efectiva de las nuevas obligaciones de las empresas requiere la instauración de autoridades de regulación que se encuentren sometidas 
exclusivamente al imperio de la ley. De otro lado, es necesario que Colombia avance hacia un Estado modesto pero eficaz, asumiendo las herramientas legales propias del derecho del compliance. En ese aspecto, las experiencias internacionales demuestran que, utilizado de manera pragmática, el derecho de los negocios puede contribuir a la realización de objetivos de interés general que el Estado solo no puede alcanzar.

De otra parte, la importancia de las nociones jurídicas de regulación y compliance insta a las empresas colombianas a adaptar su visión a los nuevos imperativos éticos, que hoy reconoce el mercado internacional. Para ello, es preciso reconocer que la responsabilidad social corporativa ha dejado de ser un tema secundario, materia de soft law, para convertirse en un asunto imperativo, impulsado tanto por los grandes Estados como por las grandes empresas alrededor del mundo. Por consiguiente, resulta necesario que las sociedades asuman seriamente las responsabilidades que se desprenden de su poder económico y de su rol central en el desarrollo de una economía sana. Las compañías deben concientizarse de aspectos como los impactos de sus actuaciones sobre los derechos fundamentales de las personas. A esos efectos resulta necesario que controlen dos áreas principales: de una parte, su ámbito interno, evitando que, amparados en el poder corporativo, sus agentes vulneren bienes protegidos jurídicamente. De otra parte, es necesario que se considere el impacto del poder empresarial en el ámbito externo, a fin de impedir que la expresión de los intereses de la empresa entre en conflicto con el interés general protegido por el derecho.

\section{Referencias}

(2013). Interbolsa: Jaramillo ratifica que pagó comisión de US\$1 millón a José Roberto Arango. El Espectador. Recuperado de https://www.elespectador. $\mathrm{com} /$ noticias/economia/interbolsa-jaramillo-ratifica-pago-

Aligica, P., \& Tarko, V. (2014). Crony capitalism: rent seeking, institutions and ideology. Kyklos, 67(2), 156-176.

Alviar, H., E Lamprea, E. (Coords.). (2015). El Estado regulador en Colombia. Bogotá: Ediciones Uniandes. 
Arroyo, L., E Nieto, A. (Eds.). (2013). El derecho penal económico en la era compliance. Valencia: Tirant lo Blanch.

Cejudo, R. (2007). Capacidades y libertad. Una aproximación a la teoría de Amartya Sen. Revista Internacional de Sociología, 65(47), 9-22.

Chambers, D., McLaughlin, P. A., \& Stanley, L. (2019). Barriers to prosperity: the harmful impact of entry regulations on income inequality. Public Choice, 180(1-2), 165-190.

Charpentier, P. (2014). La gestion du risque: de l'approche juridique à l'ébauche d'une méthodologie managériale. Revue Management Avenir, 8, 191-209.

Chevallier, J. (2004). L'état régulateur. Revue française d'administration publique, 3, 473-482.

Cordier-Palasse, B. (2016). La compliance officer: chef d'orchestre du culture change management. In A. Gaudemet (Dir.), La compliance : un nouveau monde? Aspects d'une mutation du droit (pp. 129-138). Coll. Colloques. Paris: Panthéon-Assas.

Cortina, A. (29 de noviembre de 2000). Las tres edades de la ética empresarial. El País. Recuperado de http://elpais.com/diario/2018/11/29/opinion/975452411_850215.html

Crozier, M. (2014). Etat modeste, Etat moderne. Paris: Fayard.

Eccles, R., Newquist, S., E Schatz, R. (2007). Reputation and its risks. Harvard Business Review, 85(2), 104-116.

Epron, Q. (2011). Le statut des autorités de régulation et la séparation des pouvoirs. Revue française de droit administratif, 5, 1007-1018.

Fitzgerald, V. (2004). Global financial information, compliance incentives and terrorist funding. European Journal of Political Economy, 20(2), 387-401.

Foucault, M. (1976). Historia de la sexualidad. Vol 1. La voluntad de la sexualidad. La voluntad de saber. México: Siglo XXI Editores.

Foucault, M. (2007). Nacimiento de la biopolítica: curso en el Collège de France (1978-1979). México: Fondo de Cultura Económica.

Friedman, M. (September 13, 1970). The social responsibility of business is to increase its profits. New York Times Magazine, pp. 32-33.

Frison-Roche, M.-A. (1998). Les différentes définitions de la régulation. Les Pettites Affices, 82, 5-7.

Frison-Roche, M.-A. (2001). Le droit de la régulation. Recueil Dalloz, Chroniques, 610-661.

Frison-Roche, M.-A. (2004). Définition du droit de la régulation économique. En M. A. Frison-Roche (Dir.), Les régulations économiques : légitimité et efficacité. Vol. 1 (pp. 7-15). Paris: Presses de Sciences Po et Dalloz. 
Frison-Roche, M.-A. (2016). Le droit de la compliance. Recueil Dalloz. Chron., 29 (32), 1871-1874.

Frison-Roche, M.-A. (2018). Compliance : avant, maintenant, après. En N. Borga, J.-Cl. Marin et J.-Cl. Roda, J.-Cl. (Dirs.), Compliance : l'entreprise, le régulateur et le juge (pp. 23-36). Paris: Dalloz.

Gaillard, E. (1985). Le pouvoir en droit privé. Paris: Economica.

Gaudemet, A. (Dir). (2016). La compliance: un monde nouveau? Aspects d'une mutation du droit. Paris: Panthéon-Assas.

Gélard, P. (2006). Rapport. Les autorités administratives indépendantes : évaluation d'un objet juridique non identifié. Paris: Office parlementaire d'évaluation de la législation.

Gendron, C. (2006). Codes de conduite et nouveaux mouvements socioéconomiques: la constitution d'un nouvel ordre de régulation à l'ère de la mondialisation. Revue Gestion, 31(2), 55-64.

Gómez-Aller, J. (2013). Posición de garante del compliance officer por infracción del deber de control: una aproximación tópica. En L. Arroyo \& A. Nieto (Eds.), El derecho penal económico en la era compliance (pp. 337-361). Valencia: Tirant lo Blanch.

Gonzalo, J., San-Jose, F., E Ruiz-Roqueñi, M. (2017). El moral compliance, una herramienta para facilitar la ética y la sostenibilidad en las organizaciones. Revista de Responsabilidad Social de la Empresa, 26, 15-40.

Koering-Joulin, R. (1990). La notion européenne de «tribunal indépendant et impartial» au sens de l'article 6 par. 1 de la Convention européenne de sauvegarde des droits de l'homme. Revue de science criminelle, 1, 765-780.

Lequet, P. (2017). Loi «devoir de vigilance»: de l'intérêt des normes de management des risques. Revue juridique de l'environnement, 42(4), 705-725.

Majone, G. (1996). La Communauté européenne, un État régulateur. Paris: Montchrestien.

Majone, G. (1997). From the positive to the regulatory state: causes and consequences of changes in the mode of governance. Journal of Public Policy, 17(2), 139-167.

Martín, V. (2000). Acuerdos y normas. Los códigos en la ética empresarial. Telos: Revista de Estudios Interdisciplinarios en Ciencias Sociales, 2(2), 278-283.

Millán, J. (2006). Entre el mercado y el Estado: tres décadas de reformas en el sector eléctrico de América Latina. Washington: IDB.

Missas, J. (2018). La responsabilidad penal de las personas jurídicas en Colombia. Problemáticas sobre su aplicación desde la expedición del Código Penal. Criterio Jurídico, 16(1), 69-106. 
Nielsen, V. L., E Parker, C. (2008). To what extent do third parties influence business compliance? Journal of Law and Society, 35(3), 309-340.

Nieto, A. (2013). La privatización de la lucha contra la corrupción. En L. Arroyo E A. Nieto (Eds.), El derecho penal económico en la era compliance (pp. 191210). Valencia: Tirant lo Blanch.

North, D. C., Wallis, J. J., Webb, S. B., E Weingast, B. R. (2007). Limited access orders in the developing world: a new approach to the problems of development. The World Bank.

OECD. (2014). Review of telecommunication policy and regulation in Colombia. OECD Publishing.

Requena, C. (4 de julio de 2018). La empresa, derechos humanos y compliance legal. Forbes México. Recuperado de https://www.forbes.com.mx/ empresa-derechos-humanos-y-compliance-legal/

Rifkin, J. (2001). The age of access: the new culture of hypercapitalism. New York: Penguin.

Rodríguez, M. (2015). Legal compliance: conceptualización en el marco de la regulación corporativa. En Estudios sobre el futuro Código Mercantil: libro homenaje al profesor Rafael Illescas Ortiz (pp. 935-948). Madrid: Universidad Carlos III.

Rotsch, T. (2012). Criminal compliance. InDret, (1), 1-11.

Sanclemente-Arciniegas, J. (2017). Las nociones de regulación y reglamentación en derecho económico. ¿Identidad o antagonismo? Revista Misión Jurídica, 10(13), 237-263.

Sanclemente-Arciniegas, J. (2018). Evolución conceptual del derecho económico a través de la obra de Gérard Farjat. Pensamiento Jurídico, (48), 31-58.

Sanclemente-Arciniegas, J. (2019). La independencia del regulador en Francia y en Colombia. Revista de Derecho, 51, 187-210.

Santos, B. D. S. (1987). Law: a map of misreading. Toward a postmodern conception of law. Journal of Law and Society, 14(3), 279-302.

Schiller, S. (2017). Exégèse de la loi relative au devoir de vigilance des sociétés mères et entreprises donneuses d'ordre (pp. 1052-1060). La Semaine juridique, Entreprise et Affaires.

Shleifer, A. (2012). The failure of judges and the rise of regulators. Cambridge: MIT Press.

Stigler, G. (1971). The theory of economic regulation. The Bell Journal of Economics and Management Science, 3-21.

Stone, C. (1980). The place of enterprise liability in the control of corporate conduct. Yale Law Journal, 90, 1. 
Strauss, P. (1984). The place of agencies in government: separation of powers and the fourth branch. Columbia Law Review, 84(3), 573-669.

Taboada, J. (2012). Informe sobre ciegos. Dinero. Recuperado de https://www. dinero.com/opinion/articulo/informe-sobre-ciegos/164770

Teubner, G. (2011). Self-constitutionalizing TNCs? On the linkage of "private" and "public" corporate codes of conduct. Indiana Journal of Global Legal Studies, 18(2), 617-638.

Thibierge, C. (2008). Au coeur de la norme: le tracé et la mesure. Pour une distinction entre normes et règles de droit. Archives de philosophie du droit, $51,341-371$.

Tricot, J. (2016). La conformité, outil de juridicisation de la RSE et de transformation du droit. En Martin-Chenut \& Quenaudon René, La RSE saisie par le droit. Perspectives interne et internationale (pp. 303-319). Paris: Pedone.

Turinetti, A. (2018). La normalisation: étude en droit économique. Paris: PublibookSociété écrivains. 